Cuadernos de Geografía • $105 \bullet 97-116 \bullet$ València 2020

PABLO CISNEROS ÁLVAREZa

\title{
ASCENSI DUART Y EL MAPA DE LA PARTICULAR CONTRIBUCIÓN DE LA CIUDAD DE VALENCIA (1695) DE FRANCISCO ANTONIO CASSAUS
}

\begin{abstract}
RESUMEN
El objetivo de este artículo es presentar documentación inédita sobre Ascensi Duart y el mapa Huerta, y Contribución Particular de la Ciudad de Valencia (1695) del jesuita Francisco Antonio Cassaus. Los trabajos de investigación llevados a cabo en el Arxiu Històric Municipal de València y en el Arxiu del Regne de València permiten entender el proceso de impresión del mapa de Cassaus y a Ascensi Duart, una figura que, hasta este momento, no había sido estudiada por la historiografía en profundidad y que, en parte, es una gran desconocida. En este texto se aporta información sobre Duart que ayuda a conocerle mejor y que abren nuevas e interesantes vías futuras de investigación.
\end{abstract}

Palabras clave: Cartografía; Cassaus; Duart; siglo XVII; Valencia

\section{ASCENSI DUART AND THE MAP OF THE PARTICULAR CONTRIBUTION OF THE CITY OF VALENCIA (1695) BY FRANCISCO ANTONIO CASSAUS}

\section{Abstract}

The objective of this article is to present unpublished documentation of Ascensi Duart and the map Huerta, y Contribución Particular de la Ciudad de Valencia (1695) by the jesuit Francisco Antonio Cassaus. The research carried out in the Arxiu Històric Municipal de València and in the Arxiu del Regne de València allows us to understand the process of printing the map of Cassaus and Ascensi Duart, a figure that, until now, had not been studied by the historiography in depth and that, in part, is a great unknown. This text provides information about Duart that helps to know him better and that opens up new and interesting future ways for research.

a Universidad Internacional de La Rioja (UNIR). Calle de Almansa, 101, 28040 Madrid. pablo.cisneros@unir. net. https://orcid.org/0000-0002-4875-9273

Fecha de recepción: 4/6/20. Fecha de aceptación: 27/7/20. 
Keywords: Cartography; Cassaus; Duart; XVII century; Valencia

\section{INTRODUCCIÓN}

Poco se sabe de Ascensi Duart, de quien hasta ahora se conocían datos muy limitados. El punto de partida para su estudio es su vinculación con la obra cartográfica Huerta, y Contribución Particular de la Ciudad de Valencia (1695) del jesuita Francisco Antonio Cassaus. Contrariamente, de la vida del jesuita Francisco Antonio Cassaus (1656-1699) se sabe algo más y hay estudios destacados sobre su biografía (Faus, 2009, pp. 219-240; Rosselló, 1988, pp. 177-199). No obstante, en parte, también continúa siendo desconocida. Prueba de ello es que todavía o es ignorado en diccionarios internacionales de cartógrafos (AA.VV., 1999) o no se citan todas sus obras (Martín, 2001). Se sabe que destacó en el ámbito valenciano por ser capellán, confesor y asesor del marqués de Castel Rodrigo durante varios años, coincidiendo con el periodo en el que éste fue virrey de Valencia, entre 1691 y 1695 . En estos años realizó sus obras cartográficas conocidas. La primera fue El Reyno de Valencia dividido en sus dos gobiernos (1693) (Bas, 1997, pp. 178-179; Cisneros, 2012, pp. 602-628; Faus, 2011, pp. 77-79; García, 2004, pp. 110-123; Rosselló, 1988, pp. 166 y 170-171; Vallés, 1979, pp. 17-20) y, la segunda, Huerta, y Contribución Particular de la Ciudad de Valencia (1695) (Catalá, 1999, pp. 38-39; Cisneros, 2012, pp. 629-651; Faus, 1995b, pp. 91-108; 2009, pp. 219-240; 2011, pp. 82-83; García, 1999, pp. 367-385; Ramírez, 2014, pp. 246-249; Torres, 1997, p. 46), documento que se estudia en este texto. Curiosamente, este mapa estuvo desaparecido para la historiografía desde 1932 (Almela, 1932) a 1997 (Bas, 1997, pp. 180-181) y envuelto en varias hipótesis erróneas (Faus, 2011, p. 82), quizás motivadas por el torpe descuido del grabador quien buriló 1595 en vez de 1695, hecho éste que, evidentemente, generó que se buscara información relativa a Ascensi Duart dentro de una centuria que nunca le perteneció (Vicent, 1954, p. 153). No obstante, pronto ya se consideró que era un error en las planchas. En 1831 Francisco Xavier Borrull (1745-1838) ya hablaba de la fecha de 1695 y, además, constató que tuvo un ejemplar que, ya por aquel entonces, era "rarísimo y apreciable" (Borrull, 1831, pp. 48-49). A pesar de ello, este torpe descuido del grabador despistó a muchas historiografías posteriores.

En este artículo se va a ahondar en la figura de Ascensi Duart y en sus trabajos abordados en fechas cercanas a la impresión del mapa de Cassaus. Igualmente, la documentación aportada, tanto del Arxiu Històric Municipal de València como del Arxiu del Regne de València, ayuda a comprender mejor el proceso de impresión de Huerta, y Contribución Particular de la Ciudad de Valencia y descubre a Duart como una persona destacada en el ambiente valenciano de finales del siglo XVII.

\section{DUART Y LA IMPRESIÓN DEL MAPA HUERTA, Y CONTRIBUCIÓN PARTICULAR dE la CiUdAd de VAlencia ( i695) de Cassaus}

El mapa Huerta, y Contribución Particular de la Ciudad de Valencia de Cassaus representa el territorio circundante a Valencia -que incluía los cuarteles o distritos de Russafa, Patraix, Benimaclet y Campanar (Llopis y Perdigón, 2016, pp. 32-33) - sobre el que la propia ciudad poseía jurisdicción administrativa, judicial y fiscal (Faus, 1995b; García, 1999). Constituye, además, el primer ejemplo cartográfico valen- 
ciano en donde se muestra un interés por indicar los caminos (Arciniega, 2009, p. 203) y uno de los primeros mapas que en España marcaban los límites del municipio (Edney y Sponberg, 2020, pp. 79-80).

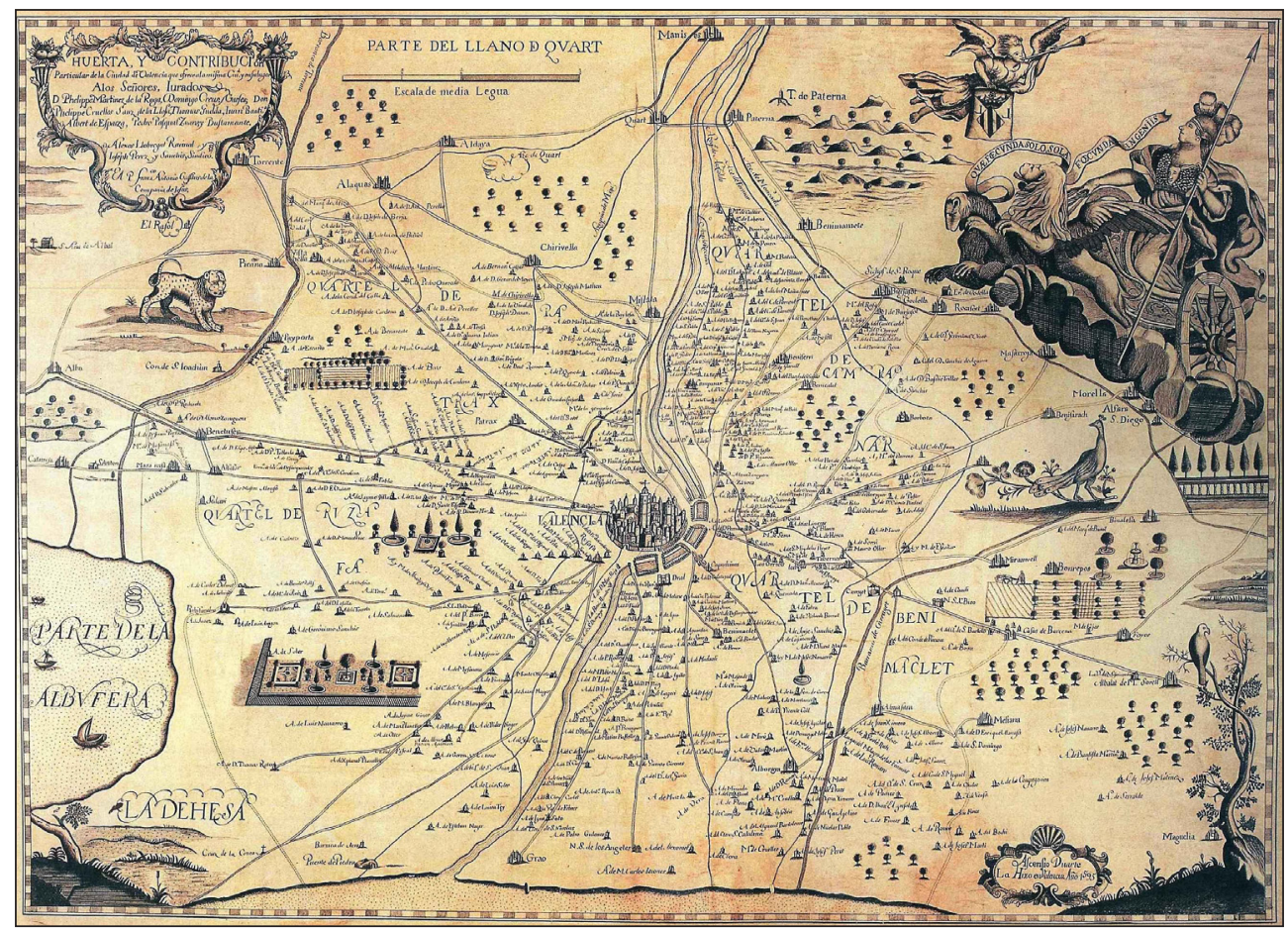

Fig. 1. Huerta, y Contribución Particular de la Ciudad de Valencia (1695), de Francisco Antonio Cassaus

Huerta, y Contribución Particular de la Ciudad de Valencia es un grabado calcográfico, de 487 x 681 mm. y de una escala aproximada de 1:23.800, que se inscribe en los márgenes habituales de la época para la representación de los términos municipales (Faus, 1995a, p. 225). En la cartela de la parte izquierda aparece escrito el siguiente texto:

Huerta, y Contribución Particular de la Ciudad de Valencia que ofrece a la misma ciudad y, en su lugar, a los señores jurados don Phelippe Martínez de la Raga, Domingo Creus Garses, don Phelippe Cruelles Sanz de la Llosa, Thomás Güelda, Juan Bautista Albert de Esparza, Pedro Pasqual Zuars y Bustamante, Alexos Llobregat, racional, y Joseph Pérez y Sanchis, síndico. El padre Francisco Antonio Casaus de la Compañía de Jesús.

En el mapa hay una cartela con roleos, hojas de acanto y coronada por una venera en su parte superior, dentro de la cual se lee “Ascencio Duarte la hizo en Valencia, año 1595”. Este, a pesar de las muchas 
hipótesis planteadas en torno a él y al fallo de impresión del año, antes mencionado, fue un librero y editor que trabajó en la Valencia de 1690 (Alexandre, 1988, 2, p. 167). En este momento, se tiene que ahondar en la relación que tiene Duart con el mapa de Cassaus. De esta vinculación se presenta documentación inédita que matiza y aporta nuevos datos relevantes que abren futuros campos de investigación.

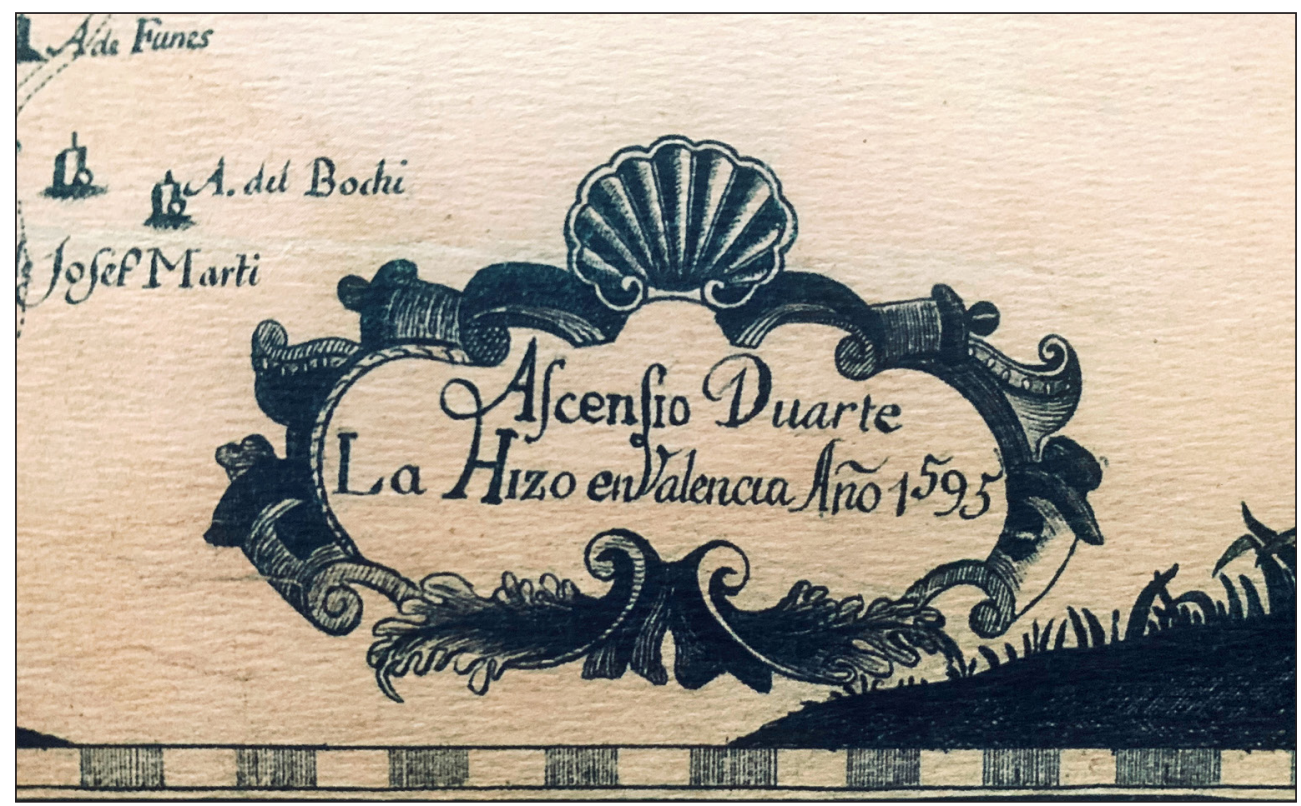

Fig. 2. Detalle de la cartela de Huerta, y Contribución Particular de la Ciudad de Valencia (1695), de Francisco Antonio Cassaus

Faus (2009, p. 225) ya encontró documentación sobre Ascensi Duart, hermano del sobrestante municipal de obras Joseph Duart. La documentación referida por Faus constataba que Duart tenía, en fechas cercanas a la realización del mapa de la Huerta, y Contribución Particular de la Ciudad de Valencia, arrendados dos cargos en el ayuntamiento. Era recaudador desde 1692 de la cisa vella de les carns y caixer de menut de la Nova Taula de Canvis ${ }^{2}$-cuatrimestre febrero-marzo de 1695-.

1 Arxiu Històric Municipal de València [en adelante AHMV]: Manual de Consells, A-223, 1691-1692, 16 y 20 de mayo de 1692, ff. 585v-586, 596v-597 y 601v-603 y también AHMV, Manual de Consells, A-227, 1694-1695, 11 de enero de 1695 , ff. $344-345 \mathrm{v}$.

2 La cisa de la carne fue uno de los impuestos indirectos que mayores ingresos daban al consistorio valenciano. Consistía en torno al $25-35 \%$ del precio final de toda la carne que se vendía en la ciudad. Su cobro era recaudado por mercaderes de prestigio y ricos comerciantes que eran partícipes de las ganancias del reembolso. Se accedía a este puesto mediante subasta pública. Por otra parte, la Nova Taula de Canvis era un banco de titularidad pública que constituía la tesorería central de la ciudad de Valencia. En esta institución financiera el caixer de menut era el organismo encargado de los pagos diarios, disponiendo de un capital que no podía superar las 6000 libras valencianas. 
El cargo de Duart en la cisa vella de les carns lo ocupó hasta el 22 de marzo de 1696 pues se ha encontrado su carta de renuncia. En ella, que figura como testigo el librero Joan Baesa -quizás sea el identificado por Serrano y Morales como Juan de Baeza (Serrano, 1897-1898, pp. 15-16)-, también presente en otros documentos firmados por Duart, se lee:

[...] en presència de l'escrivà y testimonis de sus escrits, Asensi Duart, macharrer [es uno de los términos empleados en el siglo XVII para referir al recaudador (Colomina, 1989, p. 201)] de la cisa vella de les carns, el qual dix que, per les causes y rahons assí ben vistes, renunciava, con ab tot effecte, renúncia a la dita macharra $[\ldots]^{3}$.

A pesar de que Duart renunciase en esa fecha, la documentación recoge cuentas pendientes de este cargo al menos hasta abril de $1697^{4}$. Sin embargo, sabemos que esto fue habitual. Se han encontrado también documentos sobre ajustes económicos muy tardíos sobre trabajos anteriores de Duart en la cisa vella de les carns ${ }^{5}$.

El segundo de los cargos que Ascensi Duart ocupó en el consistorio por los mismos años de la realización de Huerta, y Contribución Particular de la Ciudad de Valencia es el de caixer de menut de la Nova Taula de Canvis para el cuatrimestre febrero-marzo de 1695. Se ha encontrado que el 22 de diciembre de 1694 nombran y eligen a Ascensi Duart para el caixer de menut:

Ittem, en execució de dita convocació, [...] elegeixen y nominen en caixer de menut, per al cuadrimestre dels mesos de febrer, mars, abril y maig de l'any primer vinent 1695, a Asensi Duart, mercader, absent com si fora present, ab lo salari y emoluments ad dita caixa pertañents, donant fianses a contento de ses señories ${ }^{6}$.

Poco tiempo después, el 10 de enero de 1695 "habiliten en fianses d'Asensi Duart, mercader, per a

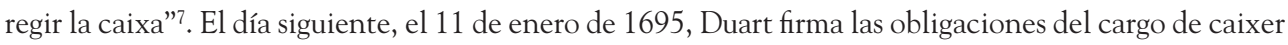
de menut de la Nova Taula de Canvis ${ }^{8}$. Sin embargo, más allá de la importancia que puede tener este documento de compromiso personal de Duart con la ciudad, interesan los testigos que acuden al acto. Gracias a eso, se sabe que Duart estuvo casado con María Manuela Matheu ya que se lee "testimonis a les dites fermes d'Asensi Duart, Maria Manuela Matheu, cònyuges".

Atendiendo a esto, no es de extrañar que Duart fuera elegido como editor del plano de Cassaus pues tenía unas más que evidentes vinculaciones con el municipio. En este sentido, fue Faus (2009) el primero en encontrar documentación sobre la impresión de Huerta, y Contribución Particular de la Ciu-

3 AHMV, Manual de Consells, A-227, 1695-1696, 22 de marzo de 1696, f. 481v.

4 AHMV, Manual de Consells, A-228, 1696-1697, 26 de abril de 1697, ff. 489v-490v.

5 AHMV, Manual de Consells, A-226, 1694-1695, 5 de febrero de 1695, ff. 379-381 y 19 de mayo de 1695, ff. 576r y $576 v$.

6 AHMV, Manual de Consells, A-226, 1694-1695, 22 de diciembre de 1694, f. 309v.

7 AHMV, Manual de Consells, A-226, 1694-1695, 10 de enero de 1695, f. 338v.

8 AHMV, Manual de Consells, A-226, 1694-1695, 10 de enero de 1695, ff. 344-345v. Este documento sí que es referido en otras publicaciones (Faus, 2009, p. 225).

9 AHMV, Manual de Consells, A-226, 1694-1695, 10 de enero de 1695, f. 345. 
dad de Valencia de Cassaus. El primer documento, no publicado por Faus, aunque sí referido, es el que relata como en el 29 de mayo de 1694 el pleno del ayuntamiento acuerda la impresión del mapa de la contribución. El coste de los gastos lo debería sufragar el depósito de los franceses, establecido durante la guerra de la Liga de Ausgburgo contra la Francia de Luís XIV. Aquí, todavía no aparece el nombre de Duart. En el documento, no transcrito por Faus, se lee:

Proveheixen que el mapa de la contribució y sisat de la present ciutat, que ha compost [...] Francisco Antoni Casaus de la compañia de Jesús, confesor de l'excel-lentísimo senor virrey, marqués de Castel Rodrigo, s'estampe y es fasa làmina; encomanando al dit magnifich sýndich el conservar aquella. Y, que el gasto que es farà, es pague dels depòsits del francesos ${ }^{10}$.

No obstante, en otros documentos municipales encontrados por Faus ya aparece el nombre de Ascensi Duart como el responsable de Huerta, y Contribución Particular de la Ciudad de Valencia ya que es a él a quien se le paga por la impresión de este trabajo. El referido investigador cita un pago a Duart del 17 de diciembre de 1694 y otro de abril de 1697 (Faus, 2009, p. 224). La consulta de esta documentación citada -tampoco trascrita- por Faus, efectivamente, refleja esos dos pagos a Duart. El primer cobro del 17 de diciembre de 1694 a Duart por Huerta, y Contribución Particular de la Ciudad de Valencia dice que:

[...] proveheixen que, dit magnifich sýndich, de dites cantitats gire, per dita Taula, a Asensi Duart, mercader, vint-y-cinch lliures a conte del treball que fe en obrir la làmina del mapa de la Contribució Particular de la present ciutat. Alsant la solta per a dit effecte [... $]^{11}$.

El segundo de los pagos efectuados a Ascensi Duart, según Faus (2009, p. 224) el resto de la cantidad que se le debía desde el primer cobro, data del 22 de abril de 1697. Faus tampoco transcribe el documento. En él se lee que:

[...] provehixen que, dit magnifich sýndich, de les cantitats, que depositen los francesos a nom de dit magnifich sýndich y a solta de dit excel-lentísimo señor virrey y dels il.lustres señors jurats. Magnifich sýndich gire, per dita Taula, a Asensi Duart, mercader, trenta lliures y nou sous per lo gasto del paper y tirar còpies del mapa que ha fet, lo dit Duart, de la Contribució Particular de la present ciutat, segons conté fermat de Claudio Bona ${ }^{12}$.

A partir de estos documentos, Faus (2009, p. 224) concluye que Ascensi Duart cobró por la impresión de Huerta, y Contribución Particular de la Ciudad de Valencia un total de 60 libras y 9 sueldos valencianos. Todo ello, como se ha expuesto, se efectuaría en dos pagos, el del 17 de diciembre de 1694 y el último del 22 de abril de 1697.

Sin embargo, sabemos que eso no fue exactamente así. Se ha encontrado en el Arxiu Històric Municipal de València otro pago efectuado a Duart entre las dos fechas citadas. El nuevo documento data del 20 de mayo de 1695, por tanto, muy cercano al primer pago. Dice así:

10 AHMV, Manual de Consells, A-225, 1693-1694, 29 de mayo de 1695, f. 637v.

11 AHMV, Manual de Consells, A-226, 17 de diciembre de 1694, f. 301 v.

12 AHMV, Manual de Consells, A-228, 1696-1697, 22 de abril de 1697, ff. 482v-483. 
Proveheixen que, dit magnifich sýndich, de les cantitats, que depositen los francesos a nom de dit magnifich sýndich y a solta de dit excel-lentísimo señor virrey y dels il.lustres señors jurats. Magnifich sýndich gire, per la Taula de València, a Asensi Duart, mercader, trenta-cinch lliures y huyt sous a compliment del treball que ha tengut en obrir la làmina del mapa de la Contribució Particular de la present ciutat. Alsant la solta per a dit effecte ${ }^{13}$.

Este texto matiza las conclusiones de Faus. Ahora habría que pensar que este documento es realmente el segundo de los pagos efectuados a Duart. Siendo así, cobraría por la impresión de Huerta, y Contribución Particular de la Ciudad de Valencia un total de 60 libras y 8 sueldos valencianos. Hay que precisar, además, que en el documento del 22 de abril de 1697 se lee "per lo gasto del paper y tirar còpies del mapa que ha fet, lo dit Duart, de la Contribució Particular de la present ciutat". De esta forma, éste debe hacer referencia a una nueva tirada posterior que se paga íntegra en ese momento y por la que cobraría 35 libras y 8 sueldos. No obstante, tampoco se debe descartar que el pago se efectuase en tercias. Siendo así, este tercer último documento de 1697 daría por concluido los plazos de pago de la estampación del mapa. Si fuese así cobraría un montante de 95 libras valencianas ${ }^{14}$ y 17 sueldos.

Gran interés tiene el documento, que se da a conocer ahora, del Arxiu Històric Municipal de València que desvela que Huerta, y Contribución Particular de la Ciudad de Valencia se debió a la iniciativa del virrey de Valencia pues es él quien, mediante un decreto del 16 de diciembre de 1694, ordena un pago del mapa. Por tanto, esta obra de Cassaus no se debe a Duart, como se había apuntado (Faus, 2009, p. 225). Además de constatar que esta obra se hizo por orden del marqués de Castel Rodrigo, el documento da a conocer que el autor de las planchas fue el calderero Miguel Sacristán, quien cobró por su trabajo catorce libras y doce sueldos valencianos. Quizás ahora, también, estemos conociendo al autor del error en la fecha del mapa, que no sería otro que el propio Sacristán. Junto a esto, igualmente, hay que suponer que el mapa ya estaba terminado a finales de 1694. Este documento dice:

Ittem, en execució de decret de l'excel-lentísimo señor virrey de Castel Rodrigo, de 16 dels presents insesatur, proveheixen que, dit magnifich sindich, de les cantitats que depositen los franceços a nom de dit magnífich síndich y a solta de dit excel-lentísimo señor virrey y dels il.lustres señors jurats y síndich, gire, per dita Taula, a Miguel Sacristán, calderer, catorse lliures y dotze sous per lo preu de la plancha que ha fet del mapa de la Contribució Particular de la present ciutat. Alsant la solta per a dit effecte ${ }^{15}$.

En el siglo XVII, el decreto "tenía un origen judicial, pero en su uso en los expedientes se empleaba como sinónimo de orden o resolución, añadiéndosele luego el término que identificaba a la entidad que la había tomado" (Lorenzo, 2006, p. 232). Por tanto, se constata que el virrey estaba detrás del mapa de Cassaus. Tras dar con esta información, se buscó el decreto del virrey en el Arxiu del Regne de València en la documentación que debía recogerlo para certificar o matizar la información del documento. Por

13 AHMV, Manual de Consells, A-226, 1694-1695, 20 de mayo de 1695, ff. 585r y 585v.

14 Como curiosidad, las libras valencianas siguen usándose, de manera testimonial, en las sentencias del Tribunal de las Aguas de Valencia, siendo el valor de la libra valenciana de 0,02€.

15 AHMV, Manual de Consells, A-226, 17 de diciembre de 1694, f. 301v. 
desgracia, no lo hallamos. Esperemos que esta aportación abra nuevos caminos e investigaciones en esta dirección. Ahora, no obstante, podemos suponer que la posibilidad de que los mapas de Cassaus del Reino de Valencia y Huerta, y Contribución Particular de la Ciudad de Valencia estuviesen vinculados, sin duda, cobra más fuerza si cabe.

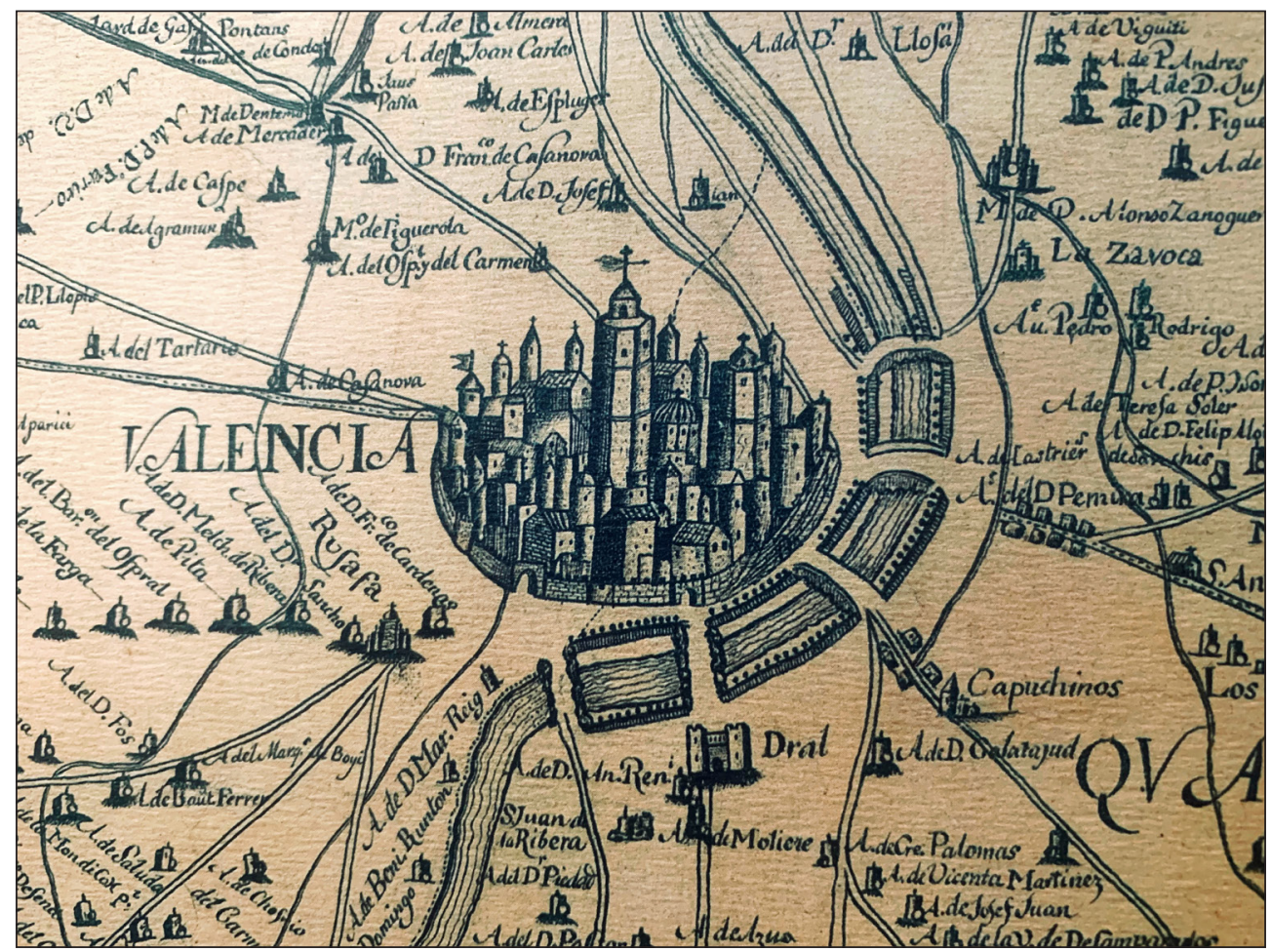

Fig. 3. Detalle de Valencia en Huerta, y Contribución Particular de la Ciudad de Valencia (1695), de Francisco Antonio Cassaus

Hay constancia documental de que, en 1712, por encargo del Ayuntamiento de Valencia, se procedió a realizar una nueva tirada de 140 ejemplares de la Huerta, y Contribución Particular de la Ciudad de Valencia de Cassaus (Faus, 2009, p. 220) ${ }^{16}$. Igualmente, la ciudad encargó a Antonio Bordázar y a Cristóbal Belda, paralelamente a la actualización que realizaron del plano de Tosca, la delineación de la Particular Contribución, que suponía la renovación de la de Cassaus. La publicación de ésta se demoró

16 Recientemente, la Societat Bibliogràłica Valenciana "Jerònima Galés” editó en 2014 una reproducción facsímil limitada a 157 ejemplares e impresa por Romeu. 
hasta 1743 (Faus, 1995a, p. 74). Del mismo modo, fue una obra que tuvo gran repercusión en proyectos cartográficos posteriores (Faus, 1995a, pp. 72-81; 1995b, pp. 91-108; 2009, pp. 229-239).

No obstante, a pesar de ello, llama la atención una anécdota dada a conocer recientemente. Germán Ramírez recoge como al poco tiempo de la invasión francesa en la ciudad en 1812, se constataba en la sesión del cabildo catedralicio del 27 de enero de 1812 que el barón Robert, general de la brigada, comandante superior y gobernador de la plaza de Valencia, solicitaba "un mapa de 1695 hecho por Ascensio Duart sobre la Huerta y Particular Contribución” pero que, finalmente, no pudieron hacerse con el (Ramírez, 2014, p. 248). No cabe duda de que este testimonio reafirma la vigencia del mapa de Cassaus, incluso muchos años después de su ejecución y a pesar de existir documentos posteriores mejorados.

No es de extrañar que los documentos demandados por el barón Robert fueran destruidos o escondidos a buen recaudo. Hay que entender lo que suponían estos documentos en el contexto que se generaron. Durante la monarquía de los Habsburgo, los reyes encargaban a sus virreyes o gobernantes mapas, cuadros, o descripciones de territorios peninsulares y del Nuevo Mundo para salvaguardar sus posesiones (Cisneros, 2019, p. 431; Fiorani, 2005; Parker, 1992; Pereda, 2007, pp. 161-182; Sanz, 2000, pp. 289-305, Woodward, 2007). Es evidente que, en el siglo XVII, centuria en el que se realiza el mapa de la contribución que se estudia, los monarcas y gobernantes llevaron a cabo una férrea política de sigilo pues eran conscientes del valor que tenía para sus adversarios y el poder que les otorgaba el conocer mejor el territorio (Hernando, 2002, p. 80). Meridianas son las palabras de Francisco de Holanda a Miguel Ángel en las que le expuso que no había más placer para los soldados que el ver "la hechura de la ciudad que han de combatir antes que la combatan" (Holanda, [1548] 1921, p. 181) o las de Pedro Rodríguez a Felipe II en las que, en 1595 dijo que los planos son "tan opuesta a los corsarios y ladrones y llave de toda esta tierra" (Cámara, 2009, p. 453). Más cercanas a la fecha de realización del mapa de Cassaus son las de Vicente Carducho en las que habla de la función de estos documentos:

[...] no se enterará por este medio del sitio y forma de una ciudad, de un castillo fuerte, de un seno y baía del mar, y de este los montes, cosa importante a las monarquías, no solo para adornar las galerías y palacios reales con semejantes pinturas, sino también en casos arduos, para que sirva a la defensa de nuestros reinos y ofensa de estraños (Carducho, [1633] 1979, p. 80).

Por tanto, hay que entender que los documentos cartográficos, y Huerta, y Contribución Particular de la Ciudad de Valencia de Cassaus no es una excepción, si caían en manos enemigas podían ser de grandísima ayuda para controlar el territorio en una situación bélica. La ciudad de Valencia, y en este caso concreto el cabildo catedralicio, actuó buscando la seguridad del municipio al "despistar", más que probable de manera intencionada, la ubicación del documento cartográfico de Cassaus. Sin duda, una historia apasionante, otra más, que encierra este mapa de finales del XVII y que, a pesar de su antigüedad y las actualizaciones posteriores, fue apreciado por las tropas napoleónicas como un valioso instrumento de control del territorio. 


\section{Duart y otros trabajos al margen del plano de la Particular contribución.}

\section{Sus trabajos para el Reino de Valencia y la ciudad de Alicante}

Teniendo en cuenta que Huerta, y Contribución Particular de la Ciudad de Valencia se debió a iniciativa del virrey, es necesario preguntarse porqué éste confió en Ascensi Duart, de quien, hasta ahora, tan solo se conocían sus trabajos municipales. Sin embargo, ahora sabemos que Duart no solo trabajó para el consistorio. La investigación en el Arxiu del Regne de València ha aportado mucha información sobre la actividad de Duart en torno a los años de realización del mapa Huerta, y Contribución Particular de la Ciudad de Valencia.

Al menos desde 1690, fecha desde la que se ha partido las investigaciones en el Arxiu del Regne de València, ya aparece Duart en la documentación. Desde esa fecha -sino antes- trabajó como alguacil del Mestre Racional. En un documento del 3 de septiembre de 1691 se ve que Duart era alguacil del Mestre Racional en 1690:

[...] lliure, per dita Taula, en virtut de dita deliberació, a Domingo Creus vint-y-quatre lliures per àpoca, 18 de julio de 1690, fermada per Asensi Duarte, alguasil del Mestre Racional $[\ldots]^{17}$.

Después, en otro documento del 29 de marzo de 1692, se vuelven a recoger los mismos trabajos:

A 29 de marc 1692. Pagui Domingo Creus vint-y-quatre liures per àpoca, de 18 de julio 1690, fermada per Asensi Duart [...] que es paguen, al dit Creus, per tantes contantes, al dit Duart, degudes de la segona tèrcia de l'any pasat de 1691 per rahó de les 12 liures que, cascun any, cobra per lo salari de son offici [alguacil del Mestre Racional] en virtut de real privilegi ${ }^{18}$.

Hay otros pagos a Duart que, si bien no se especifican a qué trabajos se refiere, constatan también sus actividades ya desde, al menos, 1690:

Dit dia [8 de marzo de 1691], a Assensi Duart noranta-sis liures, tretze sous y dos diners, per sertificatòria del Mestre Racional, de desembre $1690^{19}$.

Muy interesante es una carta que Ascensi Duart presenta el 31 de enero de 1691 al virrey. En ella, Duart, denominado librero de Valencia, cuenta el litigio que en Alicante existió con el cabildo de la ciudad por la comercialización de tres libros, cuyo privilegio de venta era desde 1686 y por un periodo de diez años. Curiosamente, la causa fue llevada por su hermano Joseph ${ }^{20}$. Con esta carta, sin duda,

17 Arxiu del Regne de València [en adelante ARV], Mestre Racional, Batlia General de València, comptes d'administració, 1691, sig. 287, f. 271.

18 ARV, Mestre Racional, Batlia General de València, corrible, 1692, sig. 373, f. 229.

19 ARV, Mestre Racional, Batlia General de València, entrades i eixides, taula, sig. 841, 8 de marzo de 1691, [s. p.].

20 Véase ARV, Real Cancilleria, epistolarium, libro 593, años 1688-1693, ff. 271-274. 
se abre la posibilidad de que Duart trabajara o tuviera vínculos profesionales también en la ciudad de Alicante. La misiva dice, en lo más sustancial, que:

[...] Por parte de Asensio Duarte, mercader de libros de la ciudad, se me ha presentado el memorial, cuia copia se os remite, en que refiere que, en el año de 1686, fui servido de prórroga al cabildo de la ciudad de Alicante, por tiempo [de] diez años. La licencia que tenía para imprimir el Arte de Antonio, Sintaxis de Torrilla y Castilla con calidad de dar, cada un año, al hospital de Aragón setenta y cinco libras y, haviendo arrendado, al dicho cabildo, el referido derecho, por dichos diez años y por precio de ciento y sesenta libras cada año, paso a imprimir y vender libros. Es, a saber, las Artes de Antonio a dos reales y medio; las Sintaxis de Torrela a real y medio y las manos de las Castillas a tres reales, que es el precio en que siempre se havían vendido y que, sin embargo, de esta antigua posesión, los jurados de esa ciudad del año 1687 [...] mandaron que no pudiesse vender dichos libros a más precio que a dos reales el Arte de Antonio, la Sintaxis a real y la mano de Castilla a dos reales. Suplicándome, por los motivos que expresa, sea servido de mandar darle permiso y facultad para que, sin embargo, o de otra provisión y de qualesquier órdenes, pueda vender dichos libros y Castilla en la forma sobredicha: como es la mano de las Castilla a tres reales, el Arte de Antonio a dos reales y medio, y la Sintasis de Torrilla a real y medio de plata durante el tiempo de dicho arrendamiento $[\ldots]^{21}$.

A partir de esta fecha, se han encontrado trabajos documentados de Duart como librero del reino. Estos consistieron, básicamente, en suministrar utensilios para escribir, diferentes libros para las actas del reino así como también reparar los libros o papeles que él mismo aportaba.

El 15 de diciembre de 1691 se libra "a Assensi Duart sexanta-dos liures, sis sous y deu diners" por sus aportaciones de "paper”, “oblees”, "plomes”, "tinta”, "aiguada de murta”, "fil de cloure cartes", "ampolles encordades", "llibre de dos mans", "un llibre de racional receptòria”, "un altre llibre receptòria general", "per lo corrible de receptòria”, "per dos llibres de deu y deix", "racional taula" y "altres dos mans de taula"22. Las contribuciones detalladas aquí serán las que, generalmente, Duart suministrará al reino.

El 31 de mayo de 1692 se le paga por los materiales de impresión y libros aportados desde el 25 de diciembre de 1691 hasta el anterior día. No obstante, no se le abona la cantidad completa de lo debido, solo 40 libras de un total de "103 liures y 16 sous"23.

Del 2 de marzo de 1693 se conservan dos documentos complementarios entre sí que refieren a un mismo pago a Duart sobre encargos de entre mayo de 1692 y junio de 1693 . Por este trabajo de librero cobró "cent once lliures, dos sous y dos diners" y consistió, sobre todo, en el aporte de material de impresión, de libros de cuentas y también "per diferents remiendos de llibres" ${ }^{24}$.

21 ARV, Real Cancilleria, epistolarium, libro n 593, años 1688-1693, ff. 270-270v.

22 ARV, Mestre Racional, Batlia General de València, comptes d'administració, 1691, sig. 287, ff. 354-354v.

23 ARV, Mestre Racional, Batlia General de València, corrible, 1692, sig. 373, ff. 301v-302.

24 ARV, Mestre Racional, Batlia General de València, comptes d'administració, 1693, sig. 289, ff. 287v-288 y ARV, Mestre Racional, Batlia General de València, corrible, 1693, sig. 374, ff. 224-224v. 
Otros dos documentos vuelven a hacer referencia a un mismo trabajo de Duart. Ambos están fechados el 15 de julio de 1693. En ellos se refleja que se le pagan "cent cinch liures, quatre sous y huit diners" por "lo recapte que a donat, a dit offici, des de 29 de janer 1693 fins 23 de juny dit any"

Por otra parte, se conservan pagos relativos a su trabajo de alguacil en el Mestre Racional. Así, hay desembolsos a Duart del 6 de julio de $1693^{26}$, del 15 del mismo mes de $1693^{27}$, del 19 de diciembre de $1693^{28}$, del 8 de enero de $1694^{29}$, del 13 de julio de $1694^{30}$, y también de un día después -este hace referencia al pago anterior- ${ }^{31}$, y del 25 de agosto de $1695^{32}$. Junto a estos, hay otros abonos efectuados a Duart en $1691^{33}, 1692^{34}, 1693^{35}, 1694^{36}, 1695^{37}, 1696^{38}$ y $1697^{39}$. Aunque no conste, muchos de ellos deben de ser atrasos o deudas de su sueldo de alguacil de Mestre Racional.

25 ARV, Mestre Racional, Batlia General de València, corrible, 1693, sig. 374, f. 226v y ARV, Mestre Racional, Batlia General de València, comptes d'administració, 1693, sig. 289, f. 290v.

26 ARV, Mestre Racional, Batlia General de València, corrible, 1693, sig. 374, f. 283.

27 ARV, Mestre Racional, Batlia General de València, entrades i eixides, esborranys, sig. 705, 15 de julio de 1693, [s. p.].

28 ARV, Mestre Racional, Batlia General de València, corrible, 1693, sig. 374, f. 283.

29 ARV, Mestre Racional, Batlia General de València, entrades i eixides, esborranys, sig. 706, 8 de enero de 1694, [s. p.] y ARV, Mestre Racional, Batlia General de València, entrades i eixides, taula, sig. 843, 8 de enero de 1694, [s. p.].

30 ARV, Mestre Racional, Batlia General de València, entrades i eixides, taula, sig. 843, 13 de julio de 1694 .

31 ARV, Mestre Racional, Batlia General de València, entrades i eixides, esborranys, sig. 707, 13 de julio de 1694, [s. p.].

32 ARV, Mestre Racional, Batlia General de València, comptes d'administració, 1695, sig. 290, f. 290.

33 ARV, Mestre Racional, Batlia General de València, entrades i eixides, taula, sig. 841, 7 de septiembre de 1691, [s. p.].

34 ARV, Mestre Racional, Batlia General de València, corrible, 1692, sig. 373, f. 229. ARV, Mestre Racional, Batlia General de València, entrades i eixides, esborranys, sig. 702, 24 de abril de 1692, [s. p.]; 30 de mayo de 1692, [s. p.]. ARV, Mestre Racional, Batlia General de València, corrible, 1692, sig. 373, f. 302r. y f. 229. ARV, Mestre Racional, Batlia General de València, corrible, 1692, sig. 373, f. 229. ARV, Mestre Racional, Batlia General de València, entrades i eixides, esborranys, sig. 703, 23 de desembre de 1692, [s. p.].

35 ARV, Mestre Racional, Batlia General de València, entrades i eixides, esborranys, sig. 705, 6 de julio de 1693, [s. p.]; 7 de julio de 1693, [s. p.]. ARV, Mestre Racional, Batlia General de València, entrades i eixides, esborranys, sig. 706, 19 de diciembre de 1693, [s. p.].

36 ARV, Mestre Racional, Batlia General de València, entrades i eixides, taula, sig. 843, 26 de abril de 1694, [s. p.] y 22 de julio de 1694, [s. p.]. ARV, Mestre Racional, Batlia General de València, entrades i eixides, esborranys, sig. 707, 22 de julio de 1694, [s. p.].

37 ARV, Mestre Racional, Batlia General de València, entrades i eixides, esborranys, sig. 710, 22 de agosto de 1695, [s. p.]. ARV, Mestre Racional, Batlia General de València, entrades i eixides, esborranys, sig. 711, 1 de diciembre de 1695 , [s. p.].

38 ARV, Mestre Racional, Batlia General de València, entrades i eixides, esborranys, sig. 714, 24 de diciembre de 1696, [s. p.].

39 ARV, Mestre Racional, Batlia General de València, entrades i eixides, taula, sig.846, 18 de marzo de 1697, [s. p.]. 


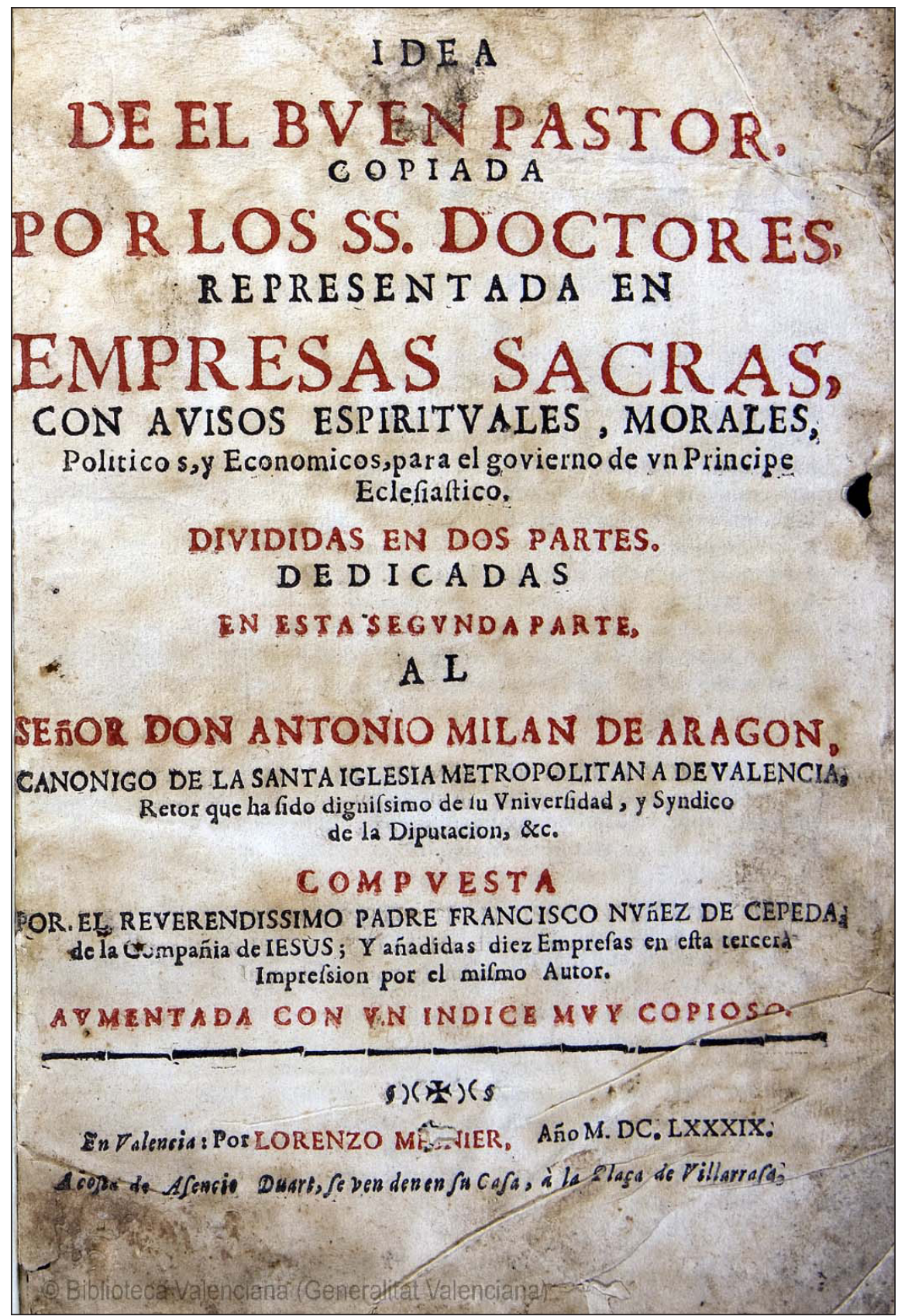

Fig. 4. Portada del libro Idea de el buen pastor copiada por los santos doctores..., de Francisco Núñez de Cepeda (Valencia, Biblioteca Valenciana, sig. XVII/912) 
En definitiva, hay que considerar a Ascensi Duart como una persona con cierto prestigio en su momento. Desempeñó cargos relevantes en el municipio de Valencia y, además, trabajó para el Mestre Racional. Junto a esto, fue librero -documentados tanto en Valencia y con vínculos en Alicante-. Desde este último trabajo, sería desde el que emprendería la edición de Huerta, y Contribución Particular de la Ciudad de Valencia de Cassaus. Aunque en el mapa se lee "Ascencio Duarte la hizo en Valencia, año 1595” hay que suponer que ésta solo se refiere a la edición ya que Cassaus fue el que delineó el mapa y ahora se sabe que Miguel Sacristán hizo las planchas.

\section{Duart y los jesuitas}

Junto a los trabajos que desempeñó Duart y desde los cuales le fue propicio editar el mapa de Cassaus, hay que destacar también el contacto que tuvo con los jesuitas. Se han encontrado obras de otros jesuitas editadas por el propio Duart y vendidas precisamente en su casa. Quizás, alguno de ellos le pudo poner en contacto con Cassaus. La primera obra que edita Duart es la del jesuita Francisco Núñez de Cepeda (1616-1690). La referencia completa de la obra dice así:

Idea de el buen pastor copiada por los santos doctores representada en empresas sacras con avisos espirituales, morales, políticos y económicos para el govierno de un príncipe eclesiástico divididas en dos partes. Dedicadas en esta segunda parte [...] compuesta por [...] Francisco Núñez de Cepeda de la Compañía de Jesús. Y añadidas diez empresas en esta tercera impresión por el mismo autor, aumentada con un índice muy copioso. En Valencia, por Lorenzo Mesnier, a costa de Asencio Duart. Se venden en su casa, a la plaça de Villarrasa, 1689.

Gracias a este libro ahora se sabe que Duart vivió en la plaza de Vilarrasa, hoy desaparecida. Estaba en frente del actual Museo Nacional de Cerámica y Artes Suntuarias “González Martî” -edificio del marqués de Dos Aguas- y desembocaba en la calle de Castelvins -hoy Poeta Querol- para morir en "Les pujades", tramo coincidente con parte de la actual calle de La Paz. Este dato podría hacer referencia a la impresión, es decir, a la vivienda o comercio de Lorenzo Mesnier -establecido en Valencia desde 1686 y hermano de Lorenzo Mesnier, activo en Murcia, Orihuela y Alicante entre, al menos, 1689 y 1727-. Pero, no obstante, fue la casa de Duart porque Mesnier se sabe que vivió "en frente de la Diputación" (Serrano, 1897-1989, p. 282). Posiblemente, en esta plaza de Vilarrasa pudo imprimirse el mapa de Cassaus y no es de extrañar que se pudiese vender. Esto era habitual en los editores de los mapas y planos en el siglo XVII. Meridiano es el testimonio de 1624 que recogió cómo en una de las propiedades que Antonio Mancelli tuvo en la villa de Madrid se podía "ber mapa de Madrid que solía estar en el portal de dicho patio colgado para venderse” (Molina, 1960, p. 226).

En la obra de Núñez de Cepeda, Duart escribe un texto -que firma como Du art-, fechado en Valencia el 29 de marzo de 1689, de cariz poético que elogia a Antonio Milán, éste lleva por título "Al señor don Antonio Milán de Aragón, canónigo de la santa iglesia metropolitana de Valencia, retor que ha sido digníssimo de su universidad y sýndico de la diputación”. Sin embargo, este texto, que aparece sin foliar, no aporta ningún dato del firmante. 


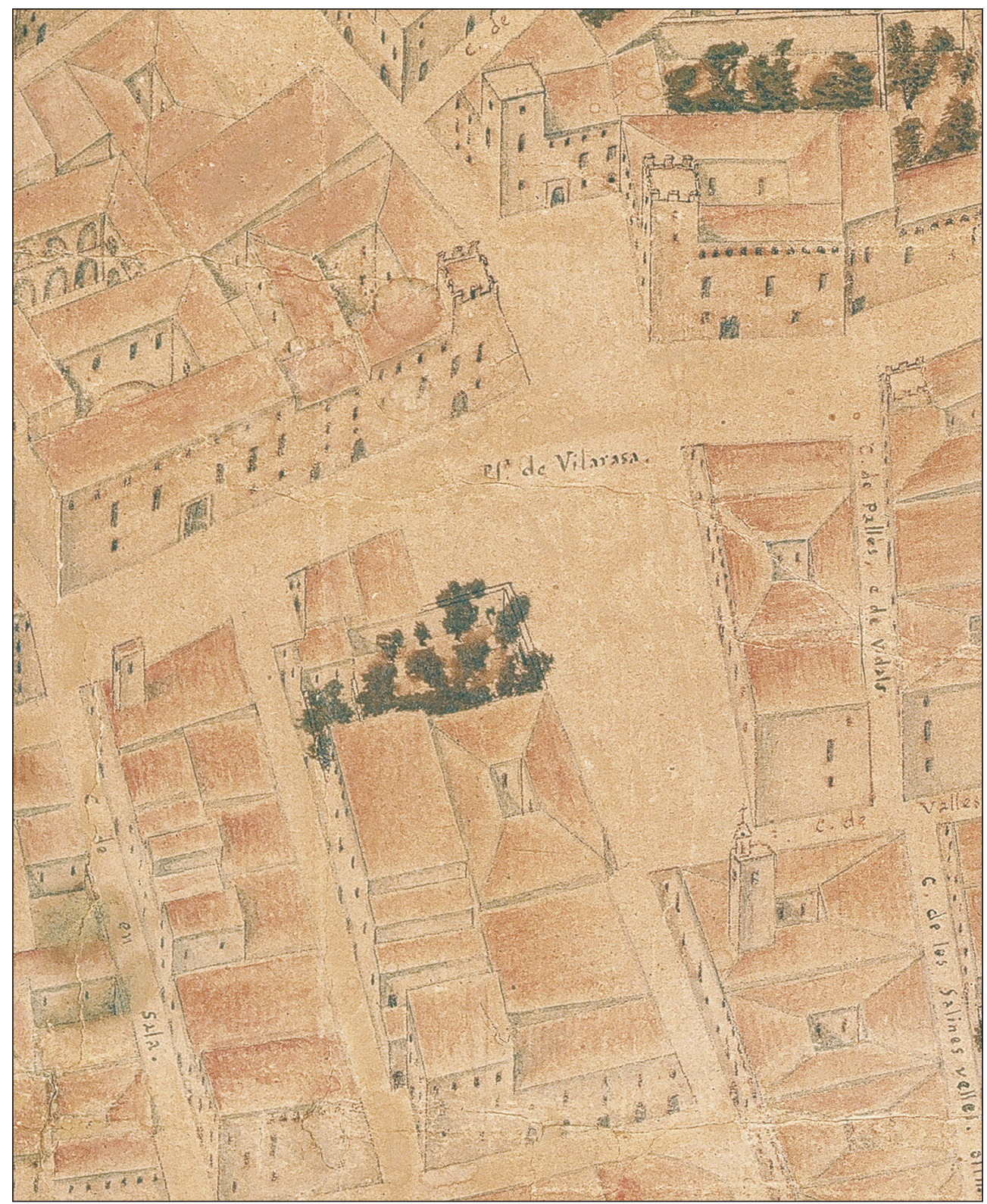

Fig. 5. Plaza Vilarrasa en el plano de Valencia de Tomás Vicente Tosca (1704) (Valencia, Museu Municipal, Ajuntament de València) 
Mayor interés tiene la otra obra editada por Duart al jesuita Francisco Garau (1640-1701), escritor y teólogo y, además, calificador del Santo Oficio. Fue una figura destacada en la comunidad pues llegó a ser catedrático del Colegio jesuita de Barcelona y rector en este último y en los colegios de Urgell, Mallorca y Zaragoza. La referencia completa de la obra es la siguiente:

El sabio instruido de la naturaleza, en quarenta máximas políticas y morales [...] por [...] Francisco Garau, de la Compañía de Jesús [...], va al fin un índice de materias predicables. En Valencia, en la imprenta de Jayme de Bordázar, a expensas de Asensio Duarte, 1690.

Esta publicación vincula a Duart con uno de los impresores más importantes del momento en la ciudad, Jaime Bordázar (Serrano, 1897-1898, pp. 25-27) padre de Antonio Bordázar. La edición de este libro constata que, al menos en 1690, Duart trabaja con Jaime Bordázar. Se ha insinuado que Huerta, y Contribución Particular de la Ciudad de Valencia se estampa, casi con toda probabilidad, en el taller de Jaime Bordázar (Faus, 2009, p. 224). Si bien no se ha encontrado documentación que certifique esta teoría, la colaboración en la obra El sabio instruido de la naturaleza de Francisco Garau entre Duart y Jaime Bordázar, al menos, testifica que esto pudo ser posible. Además, Jaime Bordázar tuvo dos de sus talleres muy cercanos a la casa de Ascensi Duart. Uno en la plaza de las Barcas y el otro, incluso más cerca, “junto a dicho Real Colegio de Corpus Christi” (Serrano, 1897-1989, p. 26).

\section{Conclusiones}

Con todo lo expuesto, y gracias a la aportación documental de este texto, habría que concluir que la edición de Huerta, y Contribución Particular de la Ciudad de Valencia se da desde varias vertientes. En primer lugar, hay que destacar que fue el virrey la persona que tuvo que impulsar la obra, pues así se desprende del decreto del 16 de diciembre de 1694. Esto, de alguna manera, permite pensar que fuese como un complemento del anterior mapa del Reino de Valencia (1693) de Cassaus. Por otra parte, habría que citar también al municipio de Valencia que, por orden del virrey, manda que se imprima y se sufraguen los gastos. Otra vertiente sería la de su autor, el jesuita Francisco Antonio Cassaus. El responsable de las planchas fue Miguel Sacristán, calderero de la ciudad. Y, el editor fue Ascensi Duart, persona que, como se ha visto, estuvo muy vinculada tanto con el entorno del virrey como con el consistorio valenciano y que patrocinó otras obras de jesuitas. Probablemente, Duart también distribuiría la obra desde su domicilio. No habría que descartar, si bien no consta que tuviese imprenta, que fuese él el tipógrafo de la obra pues pudo tener un pequeño taller en su casa de la plaza Vilarrasa. Por último, habría que mentar a Jaime Bordázar como posible impresor del mapa de la contribución de Valencia. Esto es factible pues los vínculos presentados entre el impresor y Duart existieron antes de que Cassaus empezara el mapa.

En definitiva, con este texto se ha aportado documentación sobre Ascensi Duart en fechas cercanas a Huerta, y Contribución Particular de la Ciudad de Valencia. Con esto, básicamente, se han conseguido varios objetivos. Uno es, sin duda, conocer mejor este mapa de Cassaus, auténtico referente cartográfico de la Valencia de finales del XVII. Junto a esto, se ha dado más luz a Ascensi Duart, una figura 
que todavía permanecía un tanto oculta para la historiografía. En definitiva, y por último, se sugieren nuevos y apasionantes campos de investigación de la cartografía del seiscientos.

\section{REFERENCIAS}

AA.VV. (1999): Tooley's dictionary of mapmakers. Revised edition A-D. England: Map Collector Publications.

Aleixandre, F. (1988): Libro, imprenta y censores en Valencia bajo Carlos II. En AA.VV., Homenatge al Doctor Sebastiá García Martínez (II, pp. 157-175). València: Generalitat Valenciana.

Almela, F. (1932): Alquerías de la huerta valenciana. Valencia: Monografías de Valencia Atracción. Arte y Turismo.

Arciniega, L. (2009): El saber encaminado. Caminos y viajeros por tierras valencianas de la Edad Media y Moderna. Valencia: Generalitat Valenciana.

Bas, M. (coord.) (1997): Cartografía valenciana. (siglos XIV-XIX). Valencia: Diputació Valenciana.

Borrull, F. X. (1831): Tratado de la distribución de las aguas del río Turia, y del tribunal de los acequieros de la Huerta de Valencia. Valencia: Benito Monfort.

Cámara, A. (2009): Retratos urbanos para la guerra. En V. Rodríguez/I. Rodríguez \&V. Zuriaga, El sueño de Eneas. Imágenes utópicas de la ciudad (pp. 65-88). Castellón/Valencia: UJi/Biblioteca Valenciana.

Carducho, V. ([1633] 1979) (ed. Calvo, F.): Diálogos de la pintura, su defensa, origen, esencia, definición, modos y diferencias. Madrid: Turner.

Catalá, M. Á. (1999): Valencia en el grabado. 1499-1899. Valencia: Ayuntamiento de Valencia.

Cisneros, P. (2012): La imagen grabada de la ciudad de Valencia entre 1499 y 1695 (Tesis doctoral inédita). Universitat de València, València.

Cisneros, P. (2019): Aportaciones a la biografía artística del corógrafo Antonio Mancelli: su producción americana. Archivo Español de Arte, XCII (368), 427-434. Doi: https://doi.org/10.3989/aearte.2019.27.

Colima, J. (1989): Dos vocabularis d'oficis valencians del segle XVII: G. Tarraça (1636) i V. Exulve (1643). Caplletra, 6, 179-208.

Edney, M. H. \& Sponberg, M. (2020): The History of Cartography. Volumen Four. Cartography in European Enlightenment. Chicago: University of Chicago.

Faus, A. (1995a): Mapistes. Cartografía i agrimensura a la València del segle XVIII. Valencia: Edicions Alfons el Magnàmin/Generalitat Valenciana.

Faus, A. (1995b): Demarcación y cartografía de la Particular Contribución de Valencia en el siglo XVIII. Cuadernos de Geografía, 57, 91-108.

Faus, A. (2009): El plano de la Particular Contribución de Valencia de Francisco Antonio Cassaus (1695) y sus corolarios del siglo XVIII. Cuadernos de Geografía, 86, 219-240.

Faus, A. (2011): Equívocos, mentiras, ocultaciones y medias verdades en la historia de la cartografía impresa valenciana (1584-1797). Cuadernos de Geografía, 89, 71-98. 
Fiorani, F. (2005): The marvel of maps: Art, cartography and politics in Renaissance Italy. New Haven: Yale University.

García, E. (1999): Los conflictos de jurisdicción entre Valencia y su Particular Contribución: la ciudad como parte del orden feudal vigente en la crisis del Antiguo Régimen. En E. Serrano \& E. Sarasa (coord.), Señorío y feudalismo en la Península Ibérica (s. XII-XIX) (IV, pp. 367-385). Zaragoza: Fernando el Católico.

García, V. (2004): Mapas del Reino de Valencia. De los siglos XVI a XIX. Valencia: Levante El Mercantil Valenciano.

Hernando, A. (2002): Poder, cartografía y política de sigilo en la España del siglo XVII. En F. Pereda \& F. Marías (eds.), El Atlas del rey Planeta. La descripción de España y de las costas y puertos de sus reinos de Pedro Texeira (pp. 72-97). Madrid: Nerea.

Holanda, F. de ([1548] 1921) (ed. Tormo, E.): De la pintura antigua. Madrid: Real Academia de Bellas Artes de San Fernando.

Llopis, A. \& Perdigón, L. A. (2016): Cartografía Histórica de la ciudad de Valencia (1608-1944). Valencia: Universitat Politècnica.

Lorenzo, P. L. (2006): El documento real en el siglo XVII. Algunas novedades diplomáticas. En V Jornadas Científicas sobre Documentación en España e Indias en el siglo XVII (pp. 225-238). Madrid, Universidad Complutense de Madrid.

Martín, J. (2001): Cartógrafos españoles. Madrid: Centro Nacional de Información Geográfica.

Molina, M. (1960): Planos de Madrid de los siglos XVII y XVIII. Madrid: Instituto de Estudios de Administración local.

Parker, G. (1992): Maps and minister. The Spanish Habsburg. En D. Buisseret (ed), Monarch, minister and maps. The emergence of cartography as a toll of government in Early Modern Europe (pp. 124-152). Chicago: University of Chicago.

Pereda, F. (2007): En la era de la imagen del mundo: cartografía y policía en la monarquía católica. En F. Jarauta (coord.), El mundo de los mapas (pp. 161-182). Santander: Fundación Marcelino Botín.

Ramírez, G. (2014): Huerta y contribución de la ciudad de Valencia. En Pasiones Bibliográficas: vint anys de la Societat Bibliogràfica Jerònima Galés (pp. 246-249). Valencia: Societat Bibliográfica Valenciana "Jerónima Galés".

Rosselló, V. Ma . (1988): El mapa del Regne de València de Cassaus (1693). La seua filiació i descendencia. En AA.VV., Homenatge al Doctor Sebastiá García Martínez (II, pp. 177-200). València: Generalitat Valenciana.

Rosselló, V. Ma . (2008): Cartografia Històrica dels Països Catalans. València: Universitat de València/ Institut d'Estudis Catalans.

Sanz, J. M. (2000): Las representaciones geográficas y corográficas como elementos de prestigio y representación de la monarquía: el mapa de España y las descripciones de ciudades de Enrique Cock. En E. Martínez (ed.), Actas de Congreso Madrid, Felipe II y las ciudades de la monarquía (II, pp. 289-305). Madrid, Editorial Actas. 
Serrano, J. E. (1898-1899): Reseña histórica en forma de diccionario de las imprentas que han existido en Valencia desde la introducción del arte tipográfico en España hasta el año 1868 con noticias bio-bibliográficas de los principales impresores por José Enrique Serrano y Morales. Valencia: F. Doménech.

Torres, F. (1997): La contribució de Francesc Antoni Cassaus. En Levante EMV, 24/V, 46.

Vallés, I. (1979): Cartografía histórica valenciana. Valencia: Institució Alfons el Magnànim.

Vicent, V. (1954): Bibliografía geográfica del Reino de Valencia. Zaragoza: CSIC.

Woodward, D. (ed.) (2007): Cartography in European Renaissance. Chicago/London: University of Chicago.

Cómo citar este artículo:

Cisneros Álvarez, P. (2020). Ascensi Duart y el mapa de la particular contribución de la ciudad de Valencia (1695)de Francisco Antonio Cassaus. Cuadernos de Geografía, 105, 97-116.

https://doi.org/10.7203/cguv.105.17538

\section{(C) $(1) \Theta \Theta$}

Este obra está bajo una licencia de Creative Commons Reconocimiento-NoComercial-SinObraDerivada 4.0 Internacional. 
